\title{
Permittivity Analysis of Ccto-Silicone Resin Composites
}

\author{
Sanjesh Babu ${ }^{1}$ and Pankaj Kumar ${ }^{2}$ \\ ${ }^{1}$ Department of Applied Sciences (IET), Mangalayatan University, Beswan-202145, Aligarh, (U.P.) India \\ ${ }^{2}$ Department of Chemistry, DAV (P.G.) College, Bulandshahr (U.P.) India
}

\begin{abstract}
Abtract: In this work, an attempt has been made to study permittivity of CCTO-silicone resin composites. The results show that the real permittivity was influenced by filler volume fraction. Ceramic particles produced a rise in permittivity and had more influence on samples with high volume fraction. Silicone resin had influence on the imaginary part of permittivity and generated relaxation processes on all composites. Three theoretical models were employed to predict the real permittivity of these composites, the real permittivity obtained via Maxwell-Garnett model were in close agreement with the experimental data. The real permittivity of CCTOsilicone resin composites showed weak frequency dependence at the measuring frequency range.
\end{abstract}

Key Words: CCTO, silicone resin polymer, composites, real and imaginary permittivity.

\section{Introduction}

Calcium copper titanate (CCTO) is a colossal dielectric material and has attracted a great deal of interest as a potential material for micro-electronic, capacitor and memory device (DRAM) applications [1]. In recent years, there has been an increasing interest on high dielectric constant flexible composites made up of a ferroelectric ceramic and a polymer for high density energy storage and capacitor applications [2]. However, the dielectric constant of such polymer based composites is rather low (about 50) because of the lower dielectric constant of the matrix [3-4]. For instance, in $\mathrm{BaTiO}_{3}$ /epoxy composites, though $\mathrm{BaTiO}_{3}$ has relatively high dielectric constant (>1000), the effective dielectric constant of the composite was as low as 50, even when the highest possible volume fraction of ceramics was incorporated [5]. As the volume fraction of ceramics increased, the composite, unfortunately, lost its flexibility. A new generation of ultrahigh dielectric materials such as $\mathrm{CaCu}_{3} \mathrm{Ti}_{4} \mathrm{O}_{12}$ (CCTO) can be used in order to obtain composites with better performance [6]. A number of theoretical studies and experimental observations have attempted to elucidate the remarkable dielectric properties of CCTO perovskite-like material. In this work, the dielectric performance of CCTO-silicone resin composites was studied. Relaxation phenomena as a function of frequency, temperature and filler volume fraction were analyzed.

\section{Experimental}

CCTO: silicone resin composite of 0-3 connectivity was prepared by mixing pre sintered powder of CCTO ceramic. The first set of samples were prepared in such a way that the material contains 90 percent $(90 \%)$ by volume of CCTO ceramic and 10 percent (10\%) by volume of silicone resin. A paste of the ceramic and resin is formed; one assumes that the CCTO powder has been evenly distributed into a matrix of silicone resin. To $0.5 \%$ by weight of the prepared paste, dibenzoyl peroxide was added and the paste was again mixed so that the peroxide distributes evenly throughout the volume of the mixture. Dibenzoyl Peroxide acts as a cross linking agent between the resin molecules. The paste is now injected into a steel die (mould) and the mould loaded with the paste is then heated to $140^{\circ} \mathrm{C}$. The temperature was held for 30 minutes after which the heater was turned off and the mould was allowed to cool to room temperature, opened and the material inside the cavity is removed. We obtained a cured sample which is rubber like, since the silicone resin now acts like an elastic solid, with CCTO ceramic powder distributed within the matrix like filler. This is now a 0:3 ceramic polymer composites sample. The resulting sample yields a thickness of not more than $1.5 \mathrm{~mm}$. The procedure mentioned above was repeated for samples of composition $80 \%, 70 \%, 60 \%$, and $50 \%$ by volume of CCTO.

\section{Results And Discussion}

Figure 1 shows the X-ray diffraction (XRD) pattern of the CCTO-silicone resin composites with (a) 0.8 and (b) 0.6 CCTO volume fractions. The XRD analysis for the composites revealed that the intensities of diffraction peaks were slightly increased with an increase in CCTO content in the composite. A higher percentage of silicone in the composite has a tendency to suppress the CCTO peaks. 

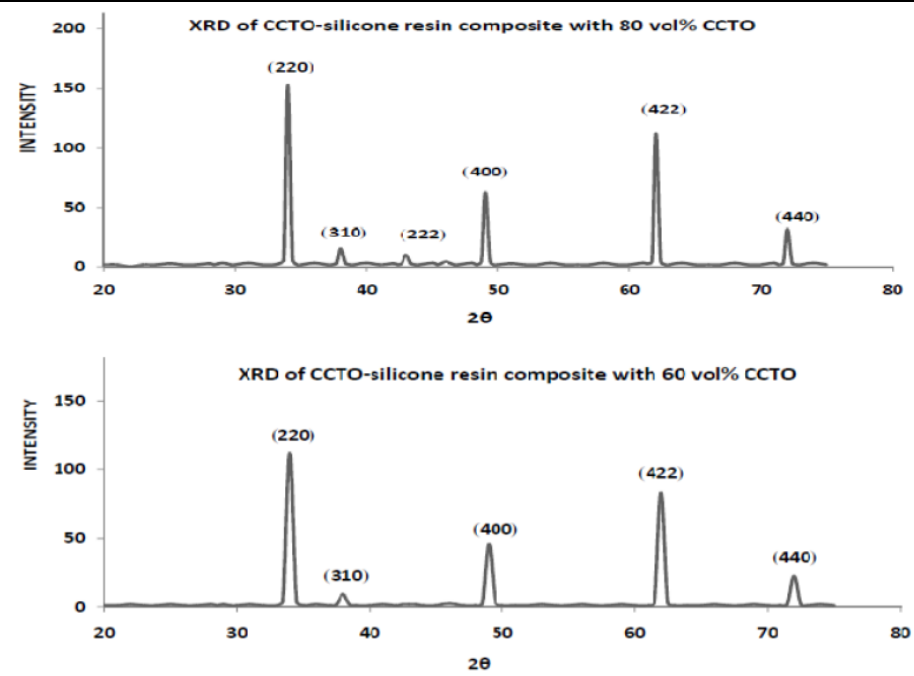

Fig. 1: XRD of composite with (a) 80 and (b) 60 vol \% CCTO
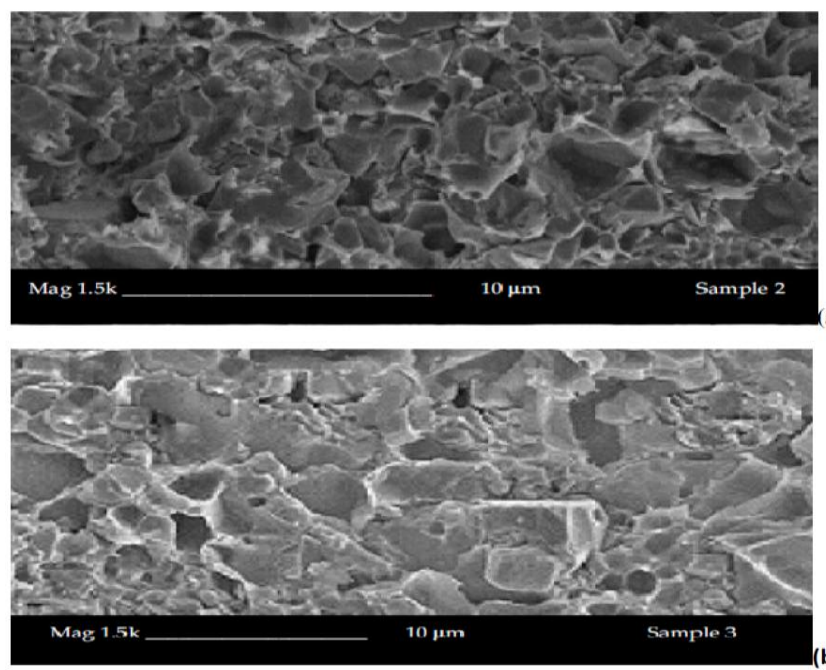

Fig. 2: SEM of composite with (a) 80 and (b) 60 vol \% CCTO

The morphology of the CCTO-silicone resin composites measured by scanning electron microscopy (SEM) is shown in Fig. 2. Figures (3-6) show real and imaginary permittivity parts as a function of frequency and temperature, respectively. As it can be expected, real permittivity rose as the ceramic volume fraction increases. It was observed that imaginary permittivity decreased at low temperatures, because of a relaxation process. Changes in the permittivity values as a function of frequency are attributed to dielectric relaxations. In order to study the frequency and temperature dependence of relaxation processes, electrical modulus was used. Figures (7-8) show the real and imaginary parts of the electrical modulus obtained through equation (1) as a function of frequency and temperature, respectively.

$M^{*}=1 / \varepsilon^{*}=1 /\left(\varepsilon^{\prime}-\varepsilon^{\prime \prime}\right)=\varepsilon^{\prime} /\left(\varepsilon^{\prime 2}+\varepsilon^{\prime 2}\right)+j \varepsilon^{\prime \prime} /\left(\varepsilon^{\prime 2}+\varepsilon^{\prime 2}\right)=M^{\prime}+j M^{\prime \prime}$

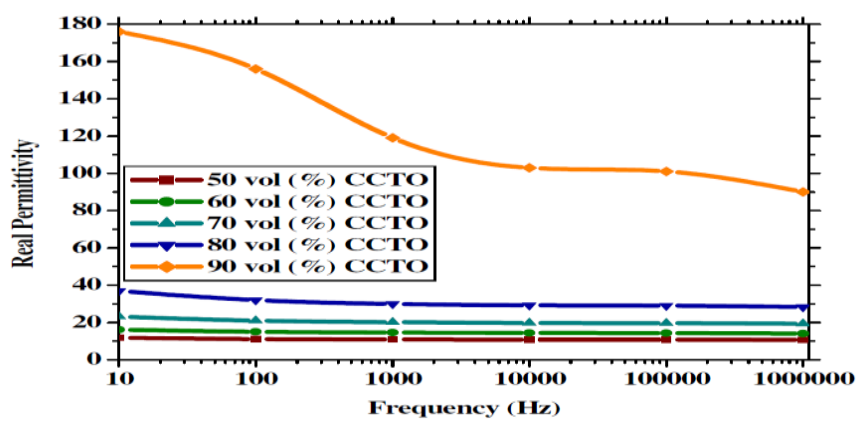

Fig-3: Real permittivity vs. frequency for composites with different CCTO volume fraction (Temp. 30 $\left.{ }^{\circ} \mathbf{C}\right)$. 


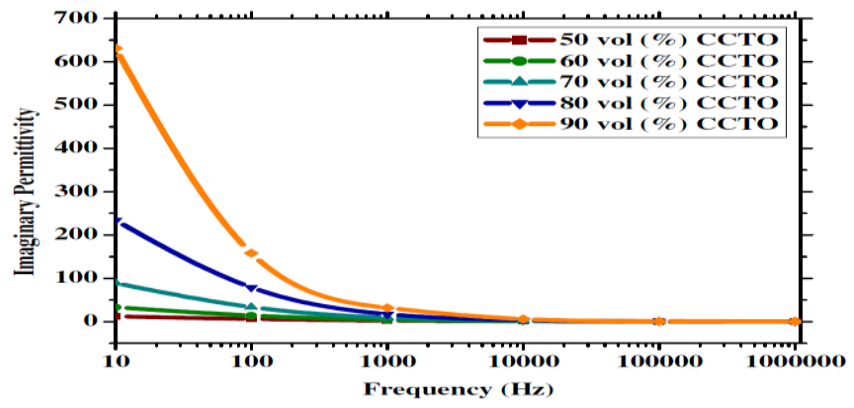

Fig-4: Imaginary permittivity vs. frequency for composites with different CCTO volume fraction (Temp. $\left.30^{\circ} \mathbf{C}\right)$.

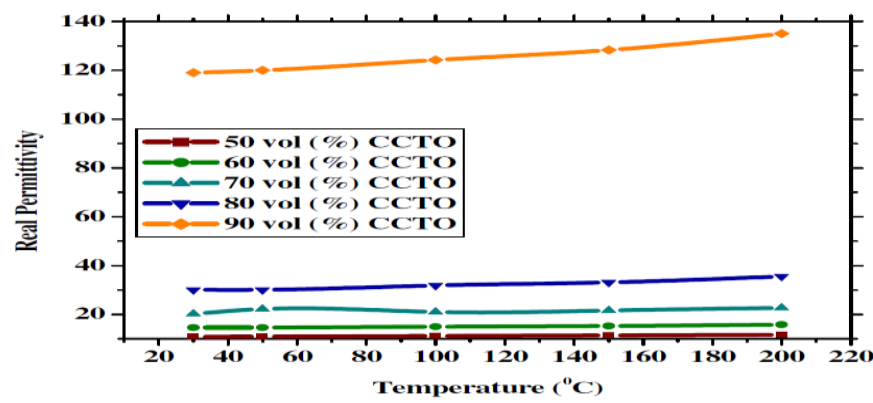

Fig-5: Real permittivity vs. temperature for composites with different CCTO volume fraction (Freq. 1000 Hz).

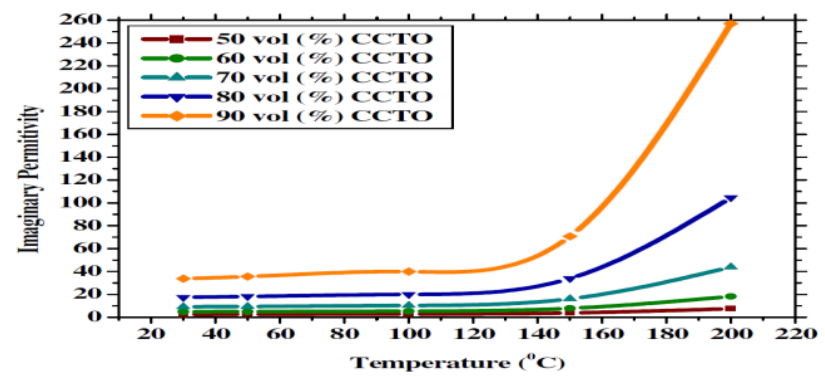

Fig-6: Imaginary permittivity vs. temperature for composites with different CCTO volume fraction (Freq. 1000 Hz).

In Figures (7-8), it can be seen that $\mathrm{M}^{\prime}$ values increased with frequency. Nevertheless, peaks in M" values were developed at this same frequency range, indicating the appearance of a relaxation process $(\alpha$ relaxation). The maximum of $\mathrm{M}^{\prime \prime}$ decreased when filler amount increased. Relaxations peaks were displaced to higher frequencies, since relaxation processes were influenced by the interfacial polarization effect which generated electric charge accumulation around the ceramic particles and the displacement of peak as the particle content increased.

A number of numerical models have been put forward by researchers to predict the real permittivity values of the composite. In the present study, the following three models were used to calculate the effective real permittivity of the CCTO-silicone resin composites.

$$
\begin{aligned}
& \log \varepsilon_{\text {eff }}^{\prime}=\varphi_{1} \log \varepsilon_{1}^{\prime}+\varphi_{2} \log \varepsilon_{2}^{\prime} \\
& \varepsilon_{\text {eff }}^{\prime}=\varepsilon_{2}^{\prime}\left[1-3 \varphi_{1}\left\{\left(\varepsilon_{1}^{\prime}-\varepsilon_{2}^{\prime}\right) /\left(\varepsilon_{1}^{\prime}+2 \varepsilon_{2}^{\prime}\right\}\right]\right. \\
& \varepsilon_{\text {eff }}^{\prime}=\varepsilon_{1}^{\prime}\left[1+\left\{3 \varphi_{1}\left(\varepsilon_{2}^{\prime}-\varepsilon_{1}^{\prime}\right)\right\} /\left\{2 \varepsilon_{1}^{\prime}+\varepsilon_{2}^{\prime}-\varphi_{1}\left(\varepsilon_{2}^{\prime} \varepsilon_{1}^{\prime}\right)\right\}\right]---(4)
\end{aligned}
$$

Equations 2-4 are the expressions of Lichtenecker model, Clausius Mossotti model and MaxwellGarnett model respectively, where $\varepsilon_{\text {eff, }}^{\prime} \varepsilon_{1}^{\prime}$ and $\varepsilon_{2}^{\prime}$ are the real permittivity of the composite, CCTO ceramic powder and silicone resin respectively and $\varphi_{1} \& \varphi_{2}$ are the volume fraction of the CCTO ceramic and the polymer, respectively. The real permittivity of silicone resin and CCTO ceramic measured at $1 \mathrm{kHz}$ and $30^{\circ} \mathrm{C}$ are 3.0 and 139, respectively. Figure 9 depicts the comparisons of real permittivity of the CCTO-silicone resin composites with the values predicted by the above equations at $1 \mathrm{kHz}$. From this figure, it is clear that the Maxwell-Garnett model is best fit of the experimental values for the CCTO-silicone resin composites. MaxwellGarnett model predict the real permittivity of the composites nearly equal to the experimental values up to 0.75 CCTO volume fractions. However, as volume fractions of CCTO increases beyond 0.75 , a deviation from the predicted value of real permittivity is observed in CCTO-silicone resin composite 


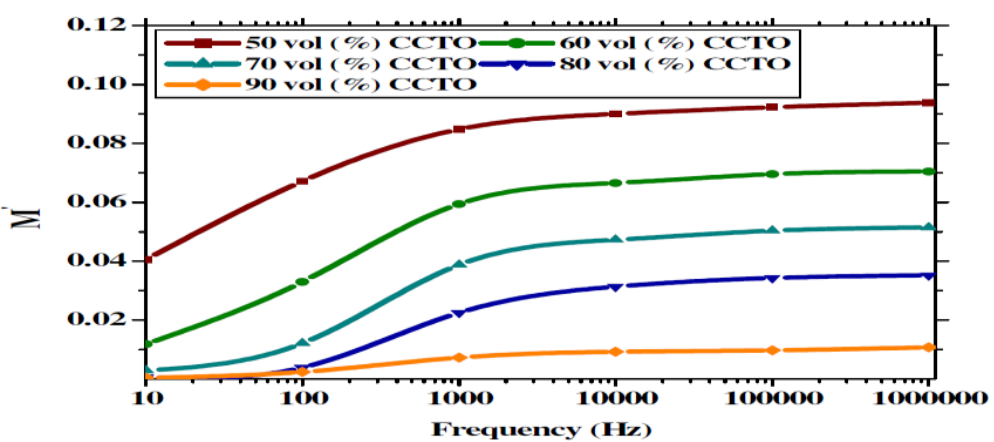

Fig-7: Real part (M') of electrical modulus vs. frequency and volume CCTO fraction (at $30^{\circ} \mathrm{C}$ ).

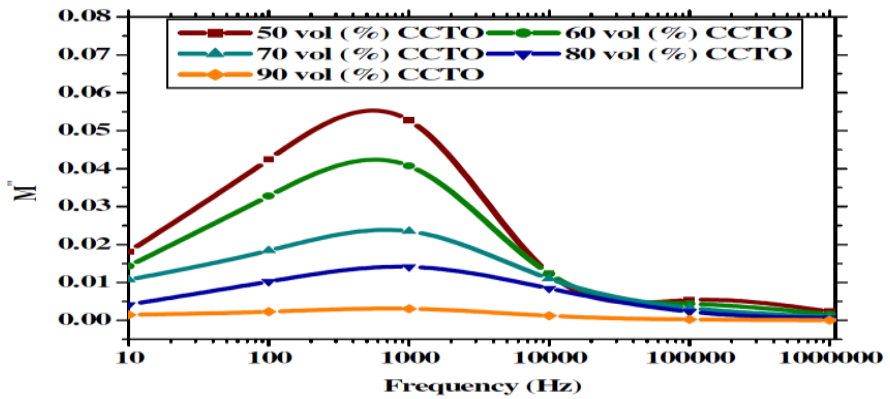

Fig-8: Imaginary part (M") of electrical modulus vs. frequency and volume CCTO fraction $\left(\mathrm{at}^{30} \mathrm{C}\right)$.

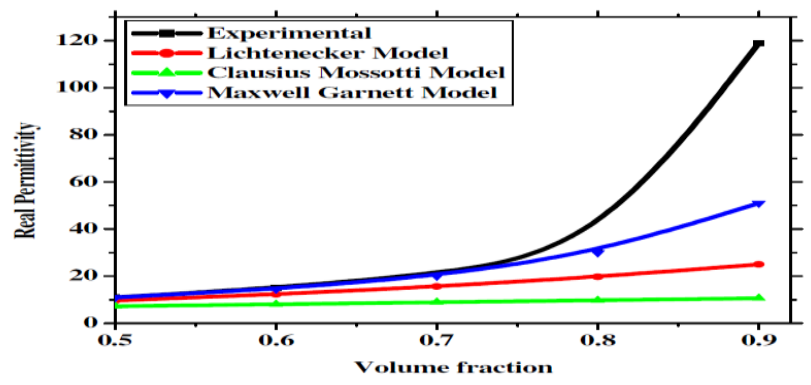

Fig-9: Real permittivity as a function of volume fraction of CCTO

\section{Conclusions}

The real permittivity was influenced by filler volume fraction. Ceramic particles produced a rise in permittivity and had more influence on samples with high volume fraction. Silicone resin had influence on the imaginary part of permittivity and generated relaxation processes on all composites. Three theoretical models were employed to predict the real permittivity of these composites, the real permittivity obtained via MaxwellGarnett model were in close agreement with the experimental data.

\section{Acknowledgments}

The authors are very grateful to the authorities of the National Physical Laboratory and the Inter University Accelerator Centre, New Delhi (India) for providing necessary facilities.

\section{References}

[1]. C. Kumar, "Dielectric properties of $\mathrm{CaCu}_{3} \mathrm{Ti}_{4} \mathrm{O}_{12}$ prepared by sol-gel self combustion technique", J Mater Sci: Mater Electron 22:579-582, 2011.

[2]. L. A. Ramajo, M.A. Ramírez, P.R. Bueno, M. M. Reboredo, M. S. Castro, "Dielectric Behaviour of $\mathrm{CaCu}_{3} \mathrm{Ti}_{4} \mathrm{O}_{12}-\mathrm{Epoxy}$ Composites", Materials Research, Vol. 11, No. 1, 85-88, 2008.

[3]. Dias CJ, Das-Gupta DK, "Inorganic ceramic/polymer ferroelectric composite electrets", IEEE Trans. Dielectr. Electr. Insul., 5(3): 706-734, 1996

[4]. Bai Y, Cheng Z-Y, Bharti V, Xu HS, Zhang QM, "High-dielectric constant ceramic-powder polymer composites", Applied Physics Letter., 25 (76): 3804-3806, 2000.

[5]. Kuo D-H, Chang C-C, Su T-Y, Wang W-K, Lin B-Y, "Dielectric behaviour of multi-doped $\mathrm{BaTiO}_{3}$-epoxy composites", Journal of the European of Ceramic Society., 21(9): 1171-1177, 2001.

[6]. Bueno PR, Ramírez MA, Varela JA, Longo E, "Dielectric spectroscopy analysis of $\mathrm{CaCu}_{3} \mathrm{Ti}_{4} \mathrm{O}_{12}$ polycrystalline systems", Applied Physics Letter., 89(19):191117-3, 2006. 